\title{
Breast Cancer Anti-Estrogen Resistance 4 Long Non-Coding RNA
}

National Cancer Institute

\section{Source}

National Cancer Institute. Breast Cancer Anti-Estrogen Resistance 4 Long Non-Coding

RNA. NCI Thesaurus. Code C132179.

Breast cancer anti-estrogen resistance 4 long non-coding RNA ( 1.3 kb) is encoded by the human BCAR4 gene. This non-coding RNA is involved in both metastasis and antiestrogen resistance in breast carcinoma. 